\title{
Immunohistochemical Evaluation of Vascular Endothelial Growth Factor (VEGF) in Splenic Hemangiomas and Hemangiosarcomas in Dogs
}

\author{
Andressa Gianotti Campos ${ }^{1}$, Juliana Alvares Duarte Bonini Campos², Daniel Soares Sanches ${ }^{3}$, \\ Maria Lúcia Zaidan Dagli ${ }^{3}$, Julia Maria Matera ${ }^{1}$ \\ ${ }^{1}$ Department of Surgery, School of Veterinary Medicine and Animal Science, University of São Paulo, São Paulo, Brazil \\ ${ }^{2}$ Department of Biostatistics and Scientific Method, School of Dentistry, São Paulo State University, Araraquara, Brazil \\ ${ }^{3}$ Department of Animal Pathology, School of Veterinary Medicine and Animal Science, University of São Paulo, São Paulo, Brazil \\ Email: gianotti@usp.br
}

Received September 21, 2012; revised October 22, 2012; accepted October 29, 2012

\begin{abstract}
Formation of new blood vessels is paramount for tumour growth and metastatic dissemination and vascular endothelial growth factor (VEGF) is one of the key regulators of this process. The purpose of this study was to evaluate the immunohistochemical expression of VEGF in 23 splenic hemangiosarcomas and 7 splenic hemangiomas in dogs. Blood tests performed previous to splenectomy were analysed for correlation with tumour VEGF expression. Results showed significantly higher VEGF expression in hemangiosarcomas than hemangiomas and lower hematocrit values and red cell count in dogs affected with malignant neoplasia $(P<0.05)$. These findings suggest the presence of high VEGF levels may be related to the malignant vascular proliferation seen in hemangiosarcomas.
\end{abstract}

Keywords: Canine; Spleen; Angiogenesis; Hemangiosarcoma; Immunohistochemistry; Vascular Endothelial Growth Factor

\section{Introduction}

Vascular endothelial growth factor (VEGF) is one of the most potent proteins involved in angiogenesis [1]. VEGF is a glycoprotein synthetized by endothelial cells, macrophages, platelets, lymphocytes, neutrophils, osteoblasts and tumoral cells $[1,2]$.

VEGF promotes proliferation, growth and migration of endothelial and inflammatory cells in response to hypoxia, inflammatory mediators or neoplasia [3-5]. It also has vasodilating effects and is 50,000 times more potent than histamine as an inducer of vascular permeability $[1,5]$.

Human and canine cancer studies show that plasma and/or tissue VEGF levels are significantly higher in cancer patients than in healthy subjects, suggesting an important role of this factor in angiogenesis and tumour growth [2,5-12].

Hemangiosarcoma (HSA) and hemangioma (HA) are malignant and benign neoplasms of vascular endothelial cells that are common in dog. Canine HSA carries an unfavourable prognosis and studies with VEGF might be applied to the detection of early lesions or identification of potential targets for the development of novel therapeutic approaches [13].
The purpose of this study was to evaluate and compare the immunohistochemical expression of vascular endothelial growth factor in splenic HA and HSA in dogs, and to correlate the results with complete blood count values obtained prior to splenectomy.

\section{Materials and Methods}

Formalin-fixed paraffin-embedded (FFPE) neoplastic tissue samples obtained from dogs presenting with splenic hemangiosarcoma or hemangioma and FFPE normal splenic tissue samples were selected for immunohistochemical analysis. Canine granulation tissue was used as a positive control for VEGF expression.

Tissue sections mounted onto salinized glass slides were deparaffinised in 2 changes of xylene (15 minutes each, in a furnace at $60^{\circ} \mathrm{C}$ ) and rehydrated through a series of descending grades of ethanol $(100 \%, 95 \%, 70 \%)$, distilled water and PBS for 5 minutes each.

Unmasking was performed in EDTA buffer for 20 minutes in microwave oven at maximum power. Endogenous peroxidase activity was blocked with a hydrogen peroxide/methanol solution for 30 minutes at room temperature in the dark. 
Following rinsing in PBS non-specific reactions were blocked with a $5 \%$ skimmed milk/distilled water solution for 15 minutes, in a furnace at $36^{\circ} \mathrm{C}$. Sectionings were then incubated with primary monoclonal mouse antihuman VEGF antibody, clone VG1 $\left(\mathrm{DAKO}^{\circledR}\right.$-code M7273) in a 1:30 dilution for 15 hours, in a moist chamber at $4^{\circ} \mathrm{C}$. The primary antibody was omitted and replaced with PBS for negative control.

Slides were incubated with Biotynilated Secondary Antibody $\left(\mathrm{DAKO}^{\circledR}\right)$ for 30 minutes, in a moist chamber at room temperature and then with Streptavin-Biotyn complex for 30 minutes at room temperature.

Slides were counterstained with hematoxilyn and eosin, washed in distilled water and mounted with coverslips in aqueous medium $\left(\mathrm{DAKO}^{\circledR}\right.$ — code $\left.\mathrm{S} 3025\right)$.

Slides were then examined under a Nikon Eclipse (E-800) microscope equipped with a video camera (Coll snap-Proc $\mathrm{cf}$ color $\left.^{\mathbb{B}}\right)$. Images were captured and analysed using Image-Pro Plus software.

The expression of VEGF by neoplastic cells was evaluated by counting the number of positive cells among at least 2000 cells in ten randomly selected fields under $20 \times$ magnification.

Data concerning laboratory workup prior to splenectomy, such as hematocrit, complete cell count, serum total protein and albumin levels were extracted from the clinical records of selected patients.

Descriptive statistics was used for data analysis. Following data testing for normality the $t$ Student test was used to compare the laboratory findings of animals affected with benign and malignant splenic neoplasia. Data homocedasticity was tested by the F-test and the $\mathrm{t}$ Student test presuming different variances was used when homocedasticity was not verified. The level of significance was set at $5 \%$.

\section{Results}

The hemangiosarcoma group of dogs comprised 13 males and 10 females aged between 8 and 14 years (mean age of 10.2 years). Affected breeds were German Shepherd $(n=8)$, Poodle $(n=3)$, Labrador Retriever $(n=$ $2)$, Weimaraner $(n=2)$, Pinscher $(n=2)$. Siberian Husky $(n=1)$, Pit Bull $(n=1)$, Schnauzer $(n=1)$, Doberman $(n$ $=1)$ and mixed breed $(n=2)$. The hemangioma group of dogs comprised 2 males and 5 females aged between 7 and 13 years (mean age of 9.5 years). This was more common in German Shepherd dogs $(n=3)$. Other breeds represented were Poodle $(n=1)$, Rotweiller $(n=1)$, Doberman $(n=1)$ and mixed breed $(n=1)$.

The mean hematocrit was $38 \%( \pm 5)$ in the hemangioma group, whereas this average was $27 \%( \pm 8)$ in the hemangiosarcoma group. Similarly, red blood cell count mean was lower in the hemangiosarcoma group than in hemangioma group (4.1 versus 5.7 millions $/ \mathrm{mm}^{3}$ ). The mean of leucocytes and platelet count were not statistically different between groups.

Immunohistochemical analysis of splenic tissues showed that all hemangiosarcoma and hemangioma endothelial neoplastic cells expressed vascular endothelial growth factor at different levels, invariably with diffuse intracitoplasmatic immunoreactivity (Figure 1). No consistent differences in staining intensity of neoplastic cells were observed between groups.

The percentage of positively stained cells was high, showing immunoreactivity in more than $50 \%$ of the cells of hemangiomas and hemangiosarcomas (Table 1).

As observed in the granulation tissue (Figure 2), intense immunoreactivity was noted in inflammatory cells (lymphocytes, neutrophils and macrophages) present in both types of neoplasia. Staining of trabecular fibroblasts was less intense.

There was a correlation between total positively labeled cells and tumor histological type. Quantification of VEGF in hemangiosarcomas was statistically higher than in hemangiomas. This findings and laboratory values of the patients studied are displayed in Table 2.

\section{Discussion}

Angiogenesis is known to play a critical role in inflammation, organ repair and tumour growth. During angiogenesis expression of the VEGF gene is induced in endothelial and inflammatory cells to stimulate VEGF secretion $[1,3]$.

Increased VEGF expression is one of the first signs of angiogenesis in tumours and contributes for malignancy characteristics such as rapid growth and development of metastasis $[1,3]$.

Uchida et al. (2008) investigated VEGF and FLT-1 receptor expression and distribution in normal canine tissues and noted that the vascular smooth muscle cells of splenic vessels stained weakly for both antibodies, while the endothelium and fibroblasts did not show sings immunohistochemical staining [14]. A small number of macrophages and lymphocytes were also positive for VEGF. The authors concluded that despite the large

Table 1. VEGF expression in hemangiosarcomas and hemangiomas, based on the percentage of positively stained cells.

\begin{tabular}{cccccc}
\hline \multicolumn{6}{c}{$\%$ of positively stained cells } \\
\hline CLASSIFICATION & $\mathrm{n}$ & $0 \dashv 25$ & $25 \dashv 50$ & $50 \dashv 75$ & $75 \dashv 100$ \\
Hemangiosarcoma & 23 & - & $2(9 \%)$ & $20(87 \%)$ & $1(4 \%)$ \\
Hemangioma & 7 & - & $1(14 \%)$ & $6(86 \%)$ & - \\
\hline
\end{tabular}


Table 2. Comparison of the laboratory characteristics of animals affected with splenic hemangioma and hemangiosarcoma.

\begin{tabular}{cccccccc}
\hline & HA & & HSA & & & \\
\hline & $\mathrm{n}$ & Mean \pm SD & $\mathrm{n}$ & Mean \pm SD & T & $P$ \\
\hline Total number of cells counted & 7 & $2433.86 \pm 340.44$ & 23 & $2997.26 \pm 939.69$ & -1.54 & 0.135 \\
VEGF+ cells & & $1390.00 \pm 150.25$ & 23 & $1847.43 \pm 631.18$ & -3.191 & $0.004^{*}$ \\
VEGF- cells & 7 & $1043.86 \pm 285.14$ & 23 & $1149.83 \pm 436.56$ & -0.6 & 0.553 \\
Hematocrit (\%) & 7 & $38.29 \pm 5.28$ & 23 & $27.35 \pm 8.64$ & 3.151 & $0.004^{*}$ \\
Red blood cells (millions $\left./ \mathrm{mm}^{3}\right)$ & 7 & $5.73 \pm 0.85$ & 23 & $4.12 \pm 1.48$ & 2.716 & $0.011^{*}$ \\
Leucocytes (milhões $/ \mathrm{mm}^{3}$ ) & 7 & $13697.71 \pm 6863.40$ & 23 & $25221.30 \pm 16705.48$ & -1.763 & 0.089 \\
Platelets (millions $/ \mathrm{mm}^{3}$ ) & 7 & $325857.14 \pm 183908.88$ & 23 & $208260.87 \pm 183336.56$ & 1.485 & 0.149 \\
\hline
\end{tabular}

at-test presuming different variances, ${ }^{*}$ statistical significance for $\alpha=0.05, \mathrm{HA}=$ hemangioma; HAS $=$ hemangiosarcoma.

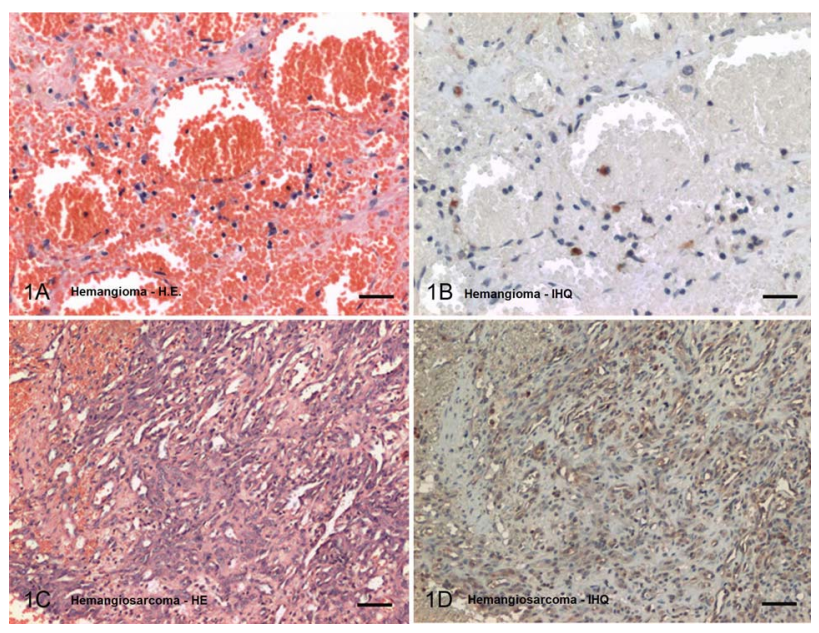

Figure. 1. Photomicrograph of canine splenic masses: (1A) Hemangioma. Large vascular space surrounded by layers of endothelial cells disposed between fine collagen septa. HE. Bar = $20 \mu \mathrm{m}$; (1B) Hemangioma. Weak cytoplasmic staining of inflammatory cells. IHQ. Bar $=20 \mu \mathrm{m}$; (1C) Hemangiosarcoma. Neoplastic endothelial cells grouped between collagen trabeculae and forming tortuous vascular channels of different seizes. HE. Bar $=40 \mu \mathrm{m}$; (1D) Hemangiosarcoma. Intense cytoplasmic staining for VEGF. IHQ. Bar $=40 \mu \mathrm{m}$.

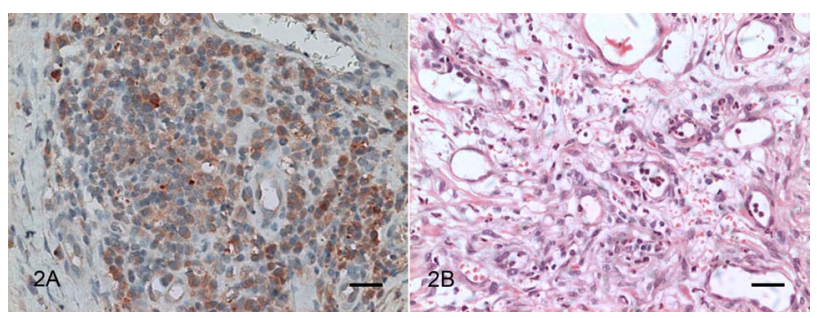

Figure 2. Photomicrograph of canine granulation tissue: (2A) Intense diffuse VEGF staining in endothelial and inflammatory cells. IHQ. Bar $=20 \mu \mathrm{m}$; (2B) Fibroplasia and immature endothelial cells surrounding newly formed blood vessels. HE. Barra $=40 \mu \mathrm{m}$. number of blood vessels in the spleen, only a small fraction of splenic cells expressed VEGF under normal conditions.

In this study similar findings were observed, with sparse macrophage and lymphocyte staining in the splenic tissue of normal dogs submitted to immunohistochemistry for VEGF. However all hemangioma and hemangiosarcoma endothelial cells showed cytoplasmic reactivity to VEGF, as indicated by the large numbers of positively stained cells.

As observed in the granulation tissue, intense staining was documented in tumour macrophages and lymphocytes. Staining was less intense in fibroblasts.

Yonemaru et al. (2006) [15] investigated VEGF in vascular tumours and documented staining in all hemangiosarcomas and hemangiomas, with higher percentage of stained cells and more intense staining in malignant than benign neoplasias.

This study supports the information presented above given not only all hemangiosarcomas but also all hemangiomas expressed VEGF. The percentage of positively stained cells was significantly higher in hemangiomas than in hemangiomas $(P<0.05)$ although precise quantification of stained cell types was not possible. Staining intensity was not evaluated and any cell showing signs of immunohistochemical staining was considered to be positive.

Histological evaluation of the spleen often reveals accumulation of intracytoplasmic pigments, particularly in the cytoplasm of macrophages. In previous studies, colour development was performed with diaminobenzidine (DAB). This compound gives both VEGF and hemosiderin a golden-brown colour, rendering differentiation difficult $[15,16]$. However, when 3-amino-9-ethylcarbazole is used in the development procedure, as in this study, differentiation from hemosiderin is unequivocal 
given a red colour is produced that is specific for the cytoplasm of anti-VEGF positive cells.

Sabattini and Bettini (2009) [13] investigated the presence of VEGF-C in 40 hemangiosarcoma and 29 hemangioma samples, both cutaneous and visceral. The authors documented weak expression in both tumour types and suggested that other growth factors may be involved in their development.

The different results documented here may reflect the different types of antibodies employed in immunohistochemistry between studies: while VEGF-A receptor VEGFR-2 is found in almost every endothelial cell, VGEF-C binds preferably to receptors expressed on lymphatic vessels (VEGFR-3).

Using in situ hybridisation Yonemaru et al. (2006) identified VEGF mRNA expression in neoplastic endothelial and inflammatory cells in hemangiosarcomas and concluded that these cells produce VEGF. However in hemangiomas only inflammatory cells expressed mRNA [15].

Studies have shown that hemangiosarcomas and hemangiomas express receptors FLT-1 and FLK-1, with higher intensity in malignant neoplasias [15]. These results suggest that the expression of vascular endothelial growth factor and its receptors may be related to the malignant proliferation observed in hemangiosarcomas.

Similarly, Sabattini and Bettini (2009) observed higher VEGFR-3 expression in malignant neoplasias although no correlation was found between VEGFR-3 and cell proliferation activity [13].

VEGF expression can also be measured in plasma or serum by ELISA. Clifford et al. (2001) compared plasma VEGF levels of 17 healthy dogs and 16 dogs affected with hemangiosarcoma and concluded that the VEGF levels were significantly higher in dogs in the neoplasia group, although this was not correlated with the stage of the disease [16].

In a prospective study, Troy et al. (2006) evaluated 44 healthy dogs, 54 dogs affected with neoplasia and 16 dogs presenting with non-neoplastic disease and documented higher serum levels of VEGF in patients in the neoplasia group [17].

In this study we chose to measure VEGF expression in tumour tissue over serum or plasma because we wanted to investigate VEGF expression specifically in the local tumour environment. The observation of high levels of VEGF in tumour tissue may support the indication of anti-VEGF drugs for cancer treatment.

Vascular endothelial growth factor regulation is related to the occurrence of hypoxia in necrotic tumour compartments and VEGF expression is high in tumours showing high degrees of necrosis. Hypoxia increases VEGF gene transcription rate and mRNA stability and may explain the higher expression of VEGF in neoplastic cells in the vicinity of necrotic areas. Also, low tissue oxygen levels act synergistically with factors such as tumoral necrosis factor $\alpha$ produced by macrophages and other inflammatory cells [4].

In this study, hematocrit values were used to assess tissue oxygenation. Our results support the conclusions by Restucci et al. (2002) given dogs with higher VEGF expression had significantly lower hematocrit values $(P$ $<0.05)[2]$.

In dogs with hemangiosarcoma, thrombocytopenia may be related to the consumption of coagulation factors secondary to intravascular disseminated coagulation. In theory, platelet destruction would elevate circulating levels of VEGF [17]. Hence a low platelet count might reflect high VEGF concentration. In this study, although VEGF was measured in tissue and not in plasma, platelet count in patients with higher VEGF expression was not significantly decreased.

\section{Conclusion}

Based on the results of this study we conclude that tumour specimens of dogs affected with splenic neoplasia express vascular endothelial growth factor (VEGF) and that staining is more intense in splenic hemangiosarcomas than hemangiomas. Low red blood cell count and hematocrit may be associated with tumoral hypoxia and higher expression of VEGF found in malignant splenic neoplasia.

\section{REFERENCES}

[1] N. Ferrara and T. Davis-Smyth, "The Biology of Vascular Endothelial Growth Factor," Endocrine Reviews, Vol. 18, No 1, 2005, pp. 4-25. doi:10.1210/er.18.1.4

[2] B. Restucci, S. Papparella, P. Maiolino and G. De Vico, "Expression of Vascular Endothelial Growth Factor in Canine Mammary Tumors," Veterinary Pathology, Vol. 39, No. 4, 2002, pp. 488-493. doi:10.1354/vp.39-4-488

[3] K. A. Houck, D. W. Leung, A. M. Bowland, J. Winer and N. Ferrara, "Dual Regulation of Vascular Endothelial Growth Factor Bioavailability by Genetic and Proteolytic Mechanisms," The Journal of Biological Chemistry, Vol. 267, No. 36, 1992, pp. 26031-26037.

[4] A. Minchenko, T. Bauer, S. Salceda and J. Caro, "Hypoxic Stimulation of Vascular Endothelial Growth Factor Expression in Vitro and in Vivo," Laboratory Investigation, Vol. 71, No. 3, 1994, pp.374-379.

[5] S. R. Platt, T. J. Scase, V. Adams, L. Wieczorek, J. Miller, F. Adamo and S. Long, "Vascular Endothelial Growth Factor Expression in Canine Intracranial Meningiomas and Association with Patient Survival," Journal of Veterinary Internal Medicine, Vol. 20, No. 3, 2006, pp. $663-$ 668. doi:10.1111/j.1939-1676.2006.tb02912.x

[6] N. Weidner, J. P. Semple and W. R. Welch, "Tumor Angiogenesis and Metastasis Correlation in Invasive Breast 
Carcinoma," New England Journal of Medicine, Vol. 324, No. 1, 1991, pp. 1-8. doi:10.1056/NEJM199101033240101

[7] E. Y. Soh, S. A. Sobhi, M. G. Wong, Y. G. Meng, A. E. Siperstein, O. H. Clark and Q. Duh, "Thyroid-Stimulating Hormone Promotes the Secretion of Vascular Endothelial Growth Factor in Thyroid Cancer Cell Lines," Surgery, Vol. 120, No. 6, 1996, pp. 944-947. doi:10.1016/S0039-6060(96)80038-9

[8] R. S. Warren, H. Yuan, M. R. Matli, N. Ferrara and D. B. Donner, "Induction of Vascular Endothelial Growth Factor by Insulin-Like Growth Factor 1 in Colorectal Carcinoma," The Journal of Biological Chemistry, Vol. 271, No. 46, 1996, pp. 29483-29488. doi: $10.1074 /$ jbc. 271.46 .29483

[9] P. Borgstrom, M. A. Bourdon, K. J. Hillan, P. Sriramarao and N. Ferrara, "Neutralizing Anti-Vascular Endothelial Growth Factor Antibody Completely Inhibits Angiogenesis and Growth of Human Prostate Carcinoma Micro Tumors in Vivo," The Prostate, Vol. 35, No. 1, 1998, pp. $1-10$.

doi:10.1002/(SICI)1097-0045(19980401)35:1<1::AID-P $\underline{\text { ROS1 } 1>3.0 . C O ; 2-O}$

[10] A. N. Al-Dissi, D. M. Haines, B. Singh and B. S. Kidney, "Immunohistochemical Expression of Vascular Endothelial Growth Factor and vascular Endothelial Growth Factor Receptor in Canine Cutaneous Fibrossarcomas," Journal of Comparative Pathology, Vol. 141, No. 4, 2009, pp. 229-236. doi:10.1016/j.jcpa.2009.05.004

[11] F. Millanta, V. Caneschi, L. Ressel, S. Citi and A. Poli, "Expression of Vascular Endothelial Growth Factor in Canine Inflammatory and Non-Inflammatory Mammary Carcinoma," Journal of Comparative Pathology, Vol. 142,
No. 1, 2010, pp. 36-42. doi:10.1016/j.jcpa.2009.06.004

[12] R. Chun and D. H. Thamm, "Targeting Angiogenesis and Tumor Vasculature," In: S. J. Withrow and D. M. Vail, Eds., Small Animal Clinical Oncology, Saunders Elsevier, St Louis, 2007, pp. 259-266.

[13] S. Sabattini and G. Bettini, "An Immunohistochemical Analysis of Canine Haemangioma and Haemangiosarcoma," Journal of Comparative Pathology, Vol. 140, No. 2-3, 2009, pp. 158-168. doi:10.1016/j.jcpa.2008.10.006

[14] N. Uchida, K. Nagai, Y. Sakurada and K. Shirota, "Distribution of Vegf and flt-1 in the Normal Dog Tissues," The Journal of Veterinary Medical Science, Vol. 70, No. 11, 2008, pp. 1273-1276. doi:10.1292/jvms.70.1273

[15] K. Yonemaru, H. Sakai, M. Murakami, T. Yanai and T. Masegi, "Expression of Vascular Endothelial Growth Factor, Basic Fibroblast Growth Factor, and Their Receptors (flt-1, flk-1, and flg-1) in Canine Vascular Tumors," Veterinary Pathology, Vol. 43, No. 6, 2006, pp. 971-980. doi:10.1354/vp.43-6-971

[16] C. A. Clifford, D. Hughes, M. W. Beal, A. J. Mackin, C. J. Henry, F. S. Shofer and K. U. Sorenmo, "Plasma Vascular Endothelial Growth Factor Concentrations in Healthy Dogs and Dogs with Hemangiosarcoma," Journal of Veterinary Medicine, Vol. 15, No. 2, 2001, pp. 131-135.

[17] G. C. Troy, W. R. Huckle, J. H. Rossmeis, D. Panciera, O. Lanz, J. L. Robertson and D. L. Ward, "Endostatin and Vascular Endothelial Growth Factor Concentrations in Healthy Dogs, Dogs with Selected Neoplasia, and Dogs with Nonneoplastic Diseases," Journal of Veterinary Internal Medicine, Vol. 20, No. 1, 2006, pp. 144-150. doi:10.1111/j.1939-676.2006.tb02834.x 